\title{
Understanding variable moment arms for the index finger MCP joints through the ACT hand
}

\author{
Ashish D. Deshpande, Ravi Balasubramanian, Ralph Lin, Brian Dellon and Yoky Matsuoka \\ addecs. washington . edu \\ University of Washington Seattle, WA, USA
}

\begin{abstract}
Human levels of dexterity has not been duplicated in a robotic form to date. Dexterity is achieved in part due to the biomechanical structure, and in part due to the neural control of movement. An anatomically correct test-bed (ACT) hand has been constructed to investigate the importance and behavioral consequences of anatomical features and neural control strategies of the human hand. This paper focused on the role of the human hand's variable moment arm. System identification was conducted on the ACT index finger's two degrees of freedom at the metacarpal-phalange (MCP) joint to provide an understanding of, for the first time, how the moment arms vary with multiple joints moving simultaneously. The specific combination of nonlinear moment arms results in an increased ability to produce force at the fingertip for the same neural input when the finger's flexion and adduction angles increase (that is toward the middle of the hand). This preliminary work will lead to answering what biomechanical and neural functions are required to construct fully dexterous robotic and prosthetic hands in the future.
\end{abstract}

\section{INTRODUCTION}

While robotic hand manipulation skills have been investigated for decades, the human level dexterity has not been implemented in a robotic hand to date. The human hand dexterity is achieved partly due to the biomechanics of the system and partly due to the neural control of movements. When human-like dexterity is realized in a robotic hand it will improve tele-operators, prosthetic hands, or also work as a test-bed for additional investigations about human hands. In this paper we present a technique to quantify the relationships between the muscle contractions and joint movements in human hands by studying these properties in a robotic hand that possesses the same biomechanics as a human hand.

We have recently completed the assembly of an anatomical robotic hand, called Anatomically Correct Test-bed (ACT) Hand (Fig.1), with the following three research goals in mind: (1) an experimental test-bed to investigate the complex neural control of human hand movements, (2) a physical prototype to test new surgical techniques for impaired hands and (3) a tele-manipulator for precision tele-operation and prosthetics. Unlike other anthropomorphic robotic hands [9], [4], [6], [17], [19], ACT Hand is anatomically correct. The local nonlinear interactions between the muscle excursions and joint movements are mimicked in the ACT Hand by bone shapes that match human bones and by a structure of tendons that connect the actuators to the finger bones. The anatomical properties allow us to implement neuromuscular

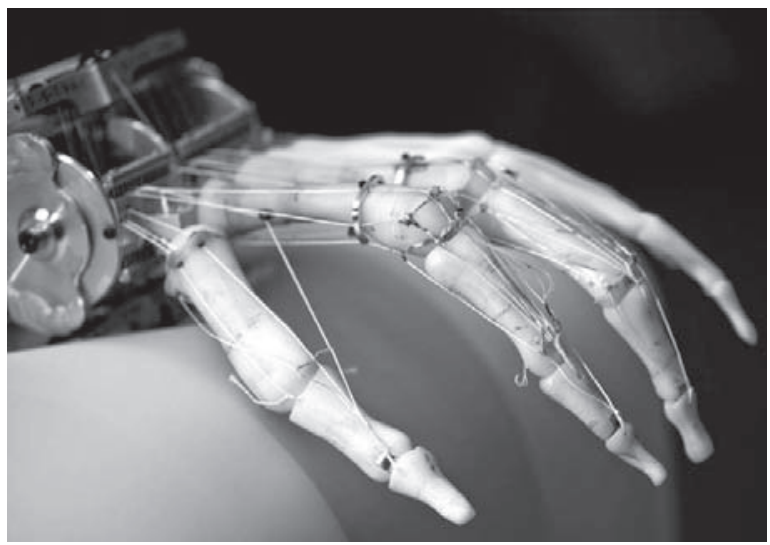

(a)
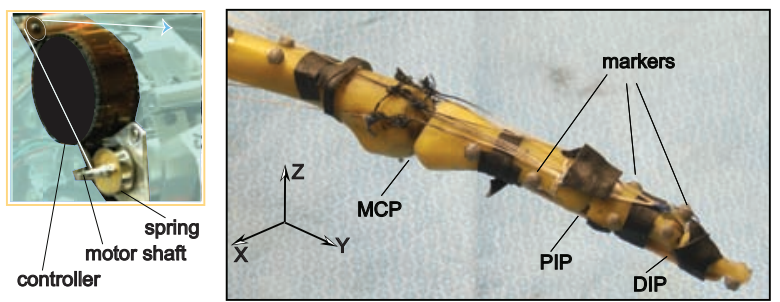

(b)

Fig. 1. Anatomically Correct Test-bed (ACT) Hand and finger. (a) The ACT Hand is modeled to be human size. The skeletal structure and tendon routing in each finger are anatomical. The hand is controlled by DC motors that are located in the forearm and connected the tendons (Photo by Ellen Garrens). (b) The ACT index finger has four anatomical degrees of freedom: DIP, PIP, and MCP with flexion/extension and abduction/adduction degrees of freedom. For the system identification, PIP and DIP joints are restricted with a splint from the bottom. The inset shows a close-up of motor shaft, loaded with a constant torque spring and back side of a miniature motor controller connected to the adjacent motor. The tendon is connected to the motor shaft and it passes over a pulley to an insertion point on a bone.

control strategies without having to compensate, in control software, for the differences in robotic hand structure and human hand anatomy.

When the robotic structure mimics the human structure, identifying robotic hardware properties and parameters can uncover interesting properties about the human system. An important characteristic of the human hand is the mechanical advantage, called as the moment arm, that each muscletendon combine has on each joint. A muscle contracts to pull on a tendon which connects to a bone and pulls the 
bone to achieve desired joint motions. The relationship between the muscle-tendon excursions and joint movements depends on the bone shapes over which tendon travels and structure of the tendon network, and is characterized by the moment arm. In case of the index finger a moment arm matrix is defined to relate excursions of seven muscles to four independent joint motions. From cadaveric and human studies it is known that the moment arm matrix is nonlinear, a function of all joint angles, and that is varies significantly from a person to another [2], [7], [13]. Since the moment arm matrix relates the muscle excursions to the joint motions and also the muscle forces to the joint torques, understanding the variations of the moment arm matrix is critical for understanding the hand control strategies and hand dexterity. Restoration of moment arm variations is considered to be an important criteria for success in hand surgeries [15] as well as in joint replacements by hand implant [26]. Moment arm matrix is studied previously for hands as well for other body parts. For example, the moment arm matrix for the elbow joint was determined by Feldman [12], and Shadmehr and Arbib developed a musculoskeletal model based on the moment arm [23]. However, exactly how the human moment arms vary with simultaneous joint movements in the human hand and how (or whether) the central nervous system (CNS) utilizes the variable moment arms to the achieve desired movement is not fully understood.

While we have reported moment arm data in the past for the ACT index finger and the ACT thumb [10], we simply used it as a tool to validate the hardware design moving one degree of freedom at a time. Moment arm data from cadaver and human subjects have also been limited to varying one joint at a time [7], [2], [13]. This paper presents the system identification technique that allows the moment arm matrix to be identified as a function of multiple moving joints for cadaveric or robotic hands. We compare our results with available cadaver data and also discuss the implications of a non-constant moment arm matrix for movement control and force generation in the hand.

\section{ACT INDEX FINGER DESCRIPTION}

In this section we present important features of the index finger of the ACT Hand which influence the moment arm determination. The details of finger skeletal structure and the tendon routing have been presented earlier in [27], [25].

\section{A. Finger Structure}

Figure 1(b) shows the ACT index finger we used for the system identification experiment. Starting from the base of the hand and working along the index finger to the tip, the finger consists of 4 bones ( 1 metacarpal, 3 phalanges) which are connected by 3 joints (MCP, PIP, DIP). All bones have nonlinear contours, same as human bone contours, to provide accurate tendon guidance. The MCP joint has two degrees of freedom, namely, adduction-abduction and flexionextension while PIP and DIP joints move in single-axis flexion-extension motions. We preserved anatomical ranges of motions for all degrees of freedom in the ACT Hand. This preservation results in a large abduction-adduction range when the finger is extended $\left( \pm 35^{\circ}\right)$ and a small range when the finger is flexed $\left( \pm 5^{\circ}\right)$ [25]. An extensor hood mechanism, which is a complex web of extensor tendons located on the dorsal side of the finger, is duplicated to match the human counterpart [27].

\section{B. Muscles and Muscle Excursions}

A human finger is controlled by 3 intrinsic muscles (PI, RI and LUM) and 4 extrinsic muscles (EDC, EIP, FDS, FDP). Due to routing of the tendons and the location of the attachment points, each muscle contributes greatly to a primary degree of freedom of the finger. For example, the FDP and FPS muscles primarily contribute to flexion, the EI and EDC muscles contribute primarily to extension. In the ACT index finger, all the same muscles are realized except for the EDC (which is equivalent to EI when only one of the fingers is controlled at a time). Each muscle is realized by a brush-less DC motor and the tendons are connected to the motor shafts after passing over pulleys. As the motor shaft rotates, the tendons slide causing an equivalent of muscle excursion.

A constant-torque spring is installed on each motor shaft which generates pre-tensions in the tendons. Each motor is connected to a miniature controller (Barrett Technology Inc [3]) with an embedded photo-sensor and an encoder wheel (with 114 ticks/deg) allowing for a high precision position sensing of the motor rotation. The controllers are connected to a RTAI Linux [22] machine which provides motor position readings at high frequency $(>500 \mathrm{~Hz})$.

\section{Moment Arm Variation}

The moment arm relates the rate of change of finger joint angles to the rate of change of muscle excursions. The moment arm varies with joint angles due to the bone shapes and tendon structure. Figure 2 illustrates three examples with varying degrees of complexity in the relationship between angle $(\theta)$ and string slide length $(L)$. In the first figure, $L=r \theta$ and moment arm, $R$, is simply $r$ which is the drum radius. In the second figure, the contour of the disk makes the relation configuration dependent and hence moment arm is $R(\theta)=\frac{\partial L}{\partial \theta}$. The third figure is an illustration of strings sliding over the 2 DOF joint, where both the bone shape and the connected tendon structure contribute to specific moment arm properties. For this figure, the 2 DOF joint is affected by two tendons that are interconnected on a contoured bone surface. The moment arm matrix is $R_{2 \times 2}$ with elements $R_{i j}=\frac{\partial L_{i}}{\partial \theta_{j}}, i, j=1,2$.

In the case of the ACT Hand index finger, the moment arm is defined by a matrix $R$ of dimension $6 \times 4$. The finger DOFs and the muscle excursions can be defined to be related by functions $f_{i}$ as follows:

$$
l_{m i}=f_{i}(\underline{\theta}) \quad i=1, \ldots, 6,
$$



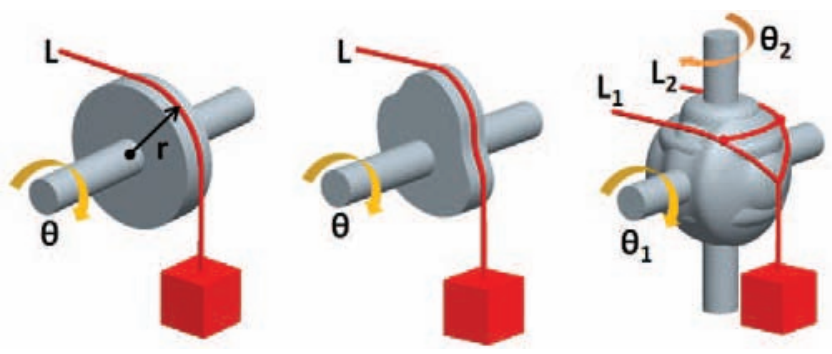

Fig. 2. Examples of moment arm variations with varying degree of complexity in the relation between angle and string slide length. For the first example moment arm, $R$ is simply $r$. For the second example moment arm is $R(\theta)=\frac{\partial L}{\partial \theta}$. For the third example moment arm matrix is $R_{2 \times 2}$ with elements $R_{i j}=\frac{\partial L_{i}}{\partial \theta_{j}}, i, j=1,2$.

where $\underline{l}_{m}$ is the vector of muscle excursions $\left(l_{m}=\right.$ $\left.\left[l_{m 1}, l_{m 2}, l_{m 3}, l_{m 4}, l_{m 5}, l_{m 6}\right]^{T}\right)$ and $\underline{\theta}$ is a vector of finger joint angles $\left(\underline{\theta}=\left[\theta_{1}, \theta_{2}, \theta_{3}, \theta_{4}\right]^{T}\right)$. Then the moment arm is defined as:

$$
\underline{l_{m}}=R \underline{\theta}
$$

where,

$R_{i j}(\theta)=\frac{\partial l_{m i}}{\partial \theta_{j}}=\frac{\partial f_{i}}{\partial \theta_{j}} \quad i=1, \ldots, 6 \quad$ and $\quad j=1, \ldots, 4$

For our system identification, we recorded muscle excursion lengths from the motor and finger angles from a motion capture system to determine the moment arm variations as functions of finger angles.

\section{Method for DATA Collection AND ANALYsis}

We have developed a system identification technique that can identify the moment arm matrix of any dimension (any number of muscles and any number of joints moving simultaneously). For this paper, we present the specific methods we used to provide results from 2 finger joint angle (MCP flexion-extension and abduction-adduction) and all 6 muscle excursions variations.

The muscle excursion data was collected by measuring the angular rotations of motors using encoders on the motor shaft. The joint angle data was collected using a motion capture system (Vicon 360). The motion capture was set up to record motions involving all four joint angles, although in the experiments for this paper, two joints were restricted with a splint. Fifteen markers were placed on the ACT finger such that marker occlusion was minimized during movements. The XYZ positions of the markers were recorded at $120 \mathrm{~Hz}$, and finger joint angles were determined by using an angle determination algorithm built into the Vicon software.

The ACT finger was then moved manually in the available range of motion of MCP abduction/adduction and flexion/extension. To ensure complete coverage, we started at the maximum MCP extension and moved the finger back and forth in the abduction/adduction plane multiple times. We repeated the same movements at multiple levels of finger flexion and this coverage procedure was repeated several times.

The muscle excursion and joint angle data were resampled at $120 \mathrm{~Hz}$ and smoothed by computing a moving average (window size 30). We computed mean muscle-length excursions for all the muscles with respect to the angles covered and fitted cubic surfaces of the form:

$$
\begin{array}{r}
l_{m i}=C_{1 i}+C_{2 i} \theta_{1}+C_{3 i} \theta_{2}+C_{4 i} \theta_{1}{ }^{2}+C_{5 i} \theta_{2}{ }^{2}+ \\
C_{6 i} \theta_{1}{ }^{2} \theta_{2}+C_{7 i} \theta_{2}{ }^{2} \theta_{1} \quad i=1, \ldots, 6
\end{array}
$$

where $C_{k i}$ are constants to be determined using a fitting algorithm. Then we computed the moment arm matrix using Equations 3 and 4.

\section{RESUlts AND VALIDATION}

\section{A. Muscle Excursions}

Table I shows muscle excursion lengths for 100 degree change in joint angle for both the ACT Hand and cadaver hands [2] (see below for more on comparison with cadaver data). Our data shows that the extrinsic muscles (FDS, FDP, and EI) show little excursion for abduction-adduction when compared to the intrinsic muscles (LUM, PI, RI). In contrast, the extrinsic muscles show large excursions for flexionextension.

Figure 3 shows example of fitted surfaces for an extrinsic muscle (FDP) and an intrinsic muscle (PI), along with the data. Mean error across all the data points was $0.85 \pm$ $0.33 \mathrm{~mm}$ which signifies that we have a close fit to the data. Thus, we have a functional mapping between joint angles and muscle excursion.

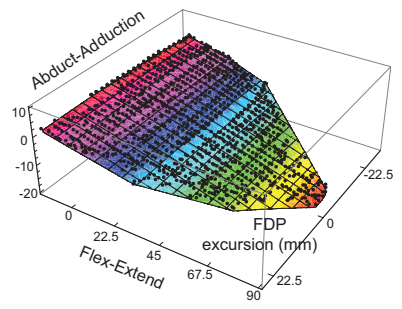

(a) FDP excursions

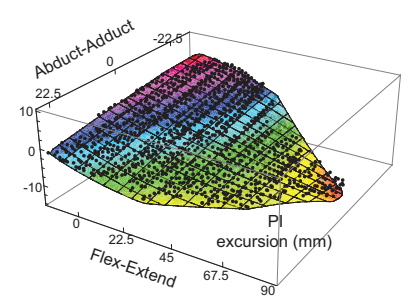

(b) PI excursions
Fig. 3. FDP and PI muscle excursions as functions of MCP angles. The cubic surfaces were fitted using the finger motion data shown in dots. The angle variations are in degrees.

\section{B. Moment Arms}

Using the above-mentioned functional relationship between joint angles and muscle excursions, we computed moment arms using Equation 3. Figure 4 shows examples of moment arms for the muscles FDP and PI. The moment arm for FDP in abduction-adduction changes sign as flexion increases, while the moment arm for FDP in flexionextension decreases as flexion increases and is symmetric about the neutral abduction angle. The moment arm for PI in abduction-adduction varies little with abduction-adduction but changes in sign as the flexion angle increases, while the 
TABLE I

MUSCLE EXCURSIONS (IN MM) FOR 100 DEGREES OF JOINT ANGLE CHANGE

\begin{tabular}{cc|c|c|c|c|c|c} 
& & EI & PI & FDP & LUM & FDS & RI \\
\hline Abduction-Adduction & ACT Hand & 2.22 & 15.35 & 2.25 & 18.65 & 1.73 & 11.65 \\
& Cadaver & 3.48 & 10.17 & 2.92 & 8.10 & 3.99 & 11.46 \\
Flexion-Extension & ACT Hand & 19.92 & 11.3 & 20.08 & 6.15 & 16.38 & 11.94 \\
& Cadaver & 15.07 & 7.87 & 18.98 & 15.26 & 21.04 & 5.88 \\
\hline
\end{tabular}

moment arm for PI in flexion-extension shows dependence on both flexion-extension and abduction-adduction angles. Table II gives the mean, maximum and minimum values of the moment arm for all six muscles. All the moment arms show large variations with respect to both MCP angles. The variations in FDP and FDS are lower than the variations in moment arms in other muscles. This may be due to the fact that the PIP and DIP joints are constrained.
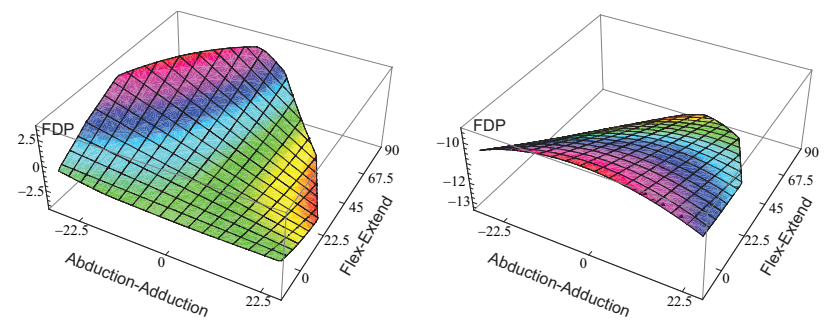

(a) FDP moment arm wrt. MCP ab- (b) FDP moment arm wrt. MCP $\mathrm{ad}$ flex-extend
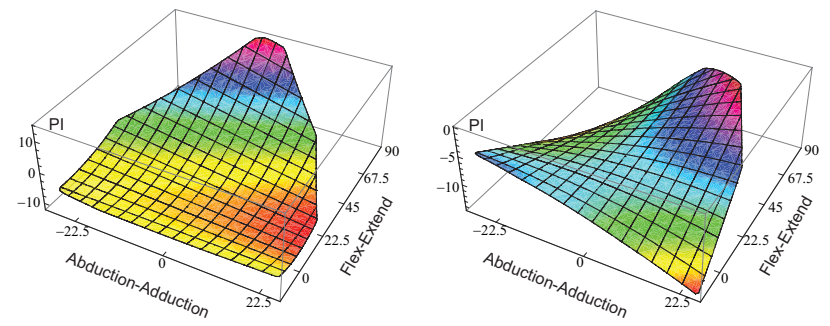

(c) PI moment arm wrt. MCP ab-ad (d) PI moment arm wrt. MCP flexexend

Fig. 4. Moment arm variations for FDP and PI muscles as functions MCP angles. The angle variations are in degrees.

In general, the moment arm in the primary direction of motion of a specific muscle increases in magnitude with the increase in primary angle. Moreover, the shape of the moment arm variation across the adduction/abduction angle does not change with the flexion angle (see Figure 4(a)). For example, the flexion moment arm magnitude for a muscle involved with flexion increases as the fingers flex, but the sign of the muscle contribution does not change. In contrast, the moment arm in the non-primary direction of motion for a muscle (i.e. adduction/abduction for FDP) changes sign as flexion angle increases (see Figure 4(b)).

\section{Validation}

The moment arm matrix determined by our method $R$ may be used to predict the joint angles given the muscle excursions using the relationship

$$
\tilde{\theta}=\int R^{-1} i d t .
$$

To validate our moment arm matrix, the ACT index finger was moved in a test path while we collected muscle excursion and joint angle data. Fingertip position was calculated using joint angles determined by using Equation 5 and also using angle data collected. Figure 5 shows the comparison of the estimated path and true fingertip path. The figure also shows a path predicted using a constant moment arm matrix $R_{c}$ measured from the ACT finger design drawings as given below

$$
R_{c}=\left[\begin{array}{ll}
0.10 & 9.80 \\
8.05 & 2.92 \\
2.97 & 6.43 \\
5.87 & 5.54 \\
1.68 & 5.59 \\
9.35 & 1.42
\end{array}\right]
$$

The path estimated using $R$ matches better with the true path $\left(r^{2}\right.$ value $\left.=0.92\right)$ when compared with the path estimated using

$R_{c}\left(r^{2}\right.$ value $\left.=0.66\right)$, and the mean error is smaller (path error using $R$ : $8.99 \pm 6.40(\mathrm{sd}) \mathrm{mm}$, and path error using $R_{c}: 48.63 \pm 25.50(\mathrm{sd}) \mathrm{mm}$ ). Note that with closedloop estimation, the potential exists to minimize path error further using $R$.

\section{Cadaver Data Comparison}

Only limited cadaver data is available for moment arm variations for single joint motions and there is no data at all involving multiple joint angle variations. We compare our results with results from [2] which is referenced and used widely in the biomechanics community. Table I shows the muscle excursions with 100 degrees of MCP joint changes for a cadaver hand and the ACT Hand. Interestingly, the trend that the extrinsic muscles show large excursion for flexion-extension and intrinsic muscle show large excursion for abduction-adduction was similar in both our data and cadaver data.

To compare with the single angle variation data [2] we generated slice plots from our varying moment arms by 
TABLE II

MOMENT ARM (IN MM) MEAN, MAX AND MIN FOR ALL SIX MUSCLES DUE TO CHANGES MCP ANGLES

\begin{tabular}{|c|ccc|ccc|}
\hline & \multicolumn{3}{|c|}{ Ab-Ad } & \multicolumn{3}{c|}{ Flex-Extend } \\
& mean & max & min & mean & max & min \\
\hline EI & 0.24 & 6.61 & -7.33 & 11.56 & 14.79 & 9.29 \\
PI & 5.80 & 14.45 & -11.28 & -7.69 & 0.02 & -14.6 \\
FDP & -0.72 & 3.23 & -4.15 & -11.18 & -9.36 & -13.34 \\
LUM & 8.03 & 11.66 & -12.01 & 2.17 & 11.29 & -7.5 \\
FDS & 1.66 & 5.64 & 0.61 & -8.50 & -5.69 & -12.41 \\
RI & 6.30 & 14.04 & -6.97 & -6.35 & 1.64 & -12.63 \\
\hline
\end{tabular}

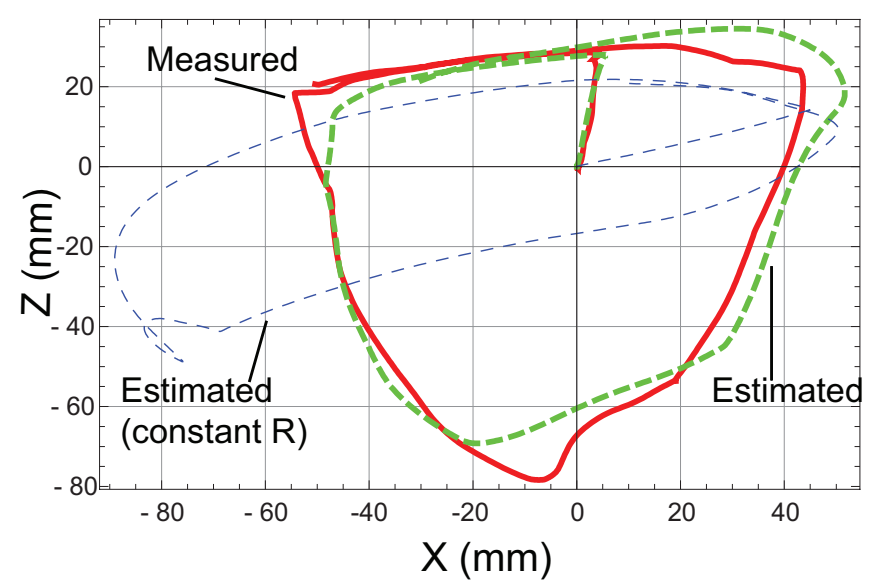

Fig. 5. A plot of finger tip X-Z position a) based on recorded joint angle data b) predicted with our method 3) predicted with constant moment arm

keeping one angle constant at a time. Figure 6 shows a comparison of the slice plots with the plots using cadaver data which are adopted from [2]. Note that the cadaveric plots were generated using data from only one female specimen. The moment arm values are higher in our case due to larger size of the ACT Hand compared to the cadaver hand. The trends for moment arm variations with flexion angle match for EI (zero slope), FDP $(-0.1 \mathrm{~mm} /$ degree slope), FDS $(-0.1 \mathrm{~mm} /$ degree slope $)$ and RI $(+0.1 \mathrm{~mm} /$ degree slope). The trends for moment arm variations with adduction angle match for PI $(+0.05 \mathrm{~mm} /$ degree slope $)$, FDP $(+0.05 \mathrm{~mm} /$ degree slope $)$, FDS $(+0.05 \mathrm{~mm} /$ degree slope $)$ and RI $(+0.05 \mathrm{~mm} /$ degree slope $)$. The differences in variations in LUM and UI with flexion angle and LUM and EI with adduction might have arisen due to differences in the structure of LUM in the cadaver hands and the ACT Hand. The ACT lumbrical tendon and associated motor is attached to the equivalent of a skeletal anchor point. In contrast, the lumbrical tendon and associated muscle in human hands are attached to another sliding tendon.

\section{Discussion}

As tendons can only pull, the tendon can only affect motion in a single direction. However, as the sign of the moment arm changes, tension on the tendon reverses the

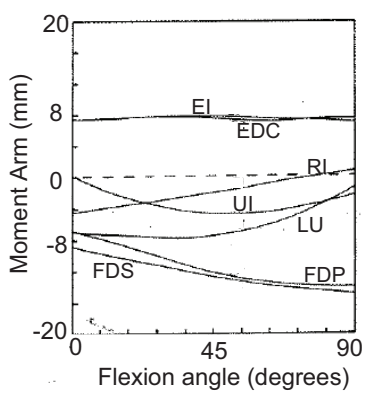

(a) Cadaver

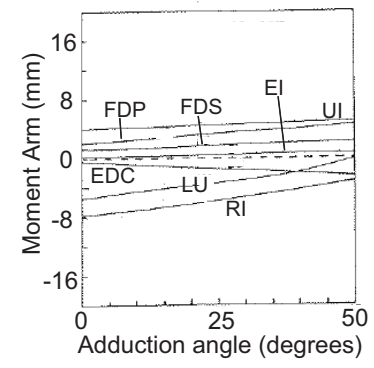

(c) Cadaver

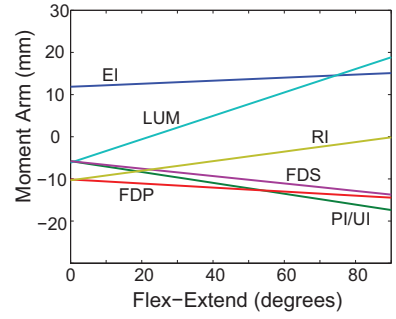

(b) ACT Hand

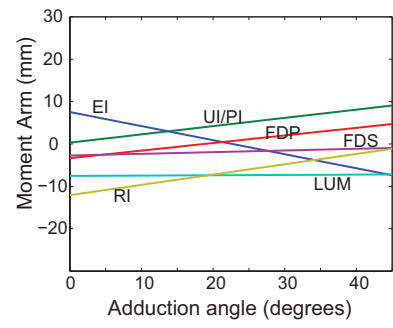

(d) ACT Hand
Fig. 6. Variations of moment arm as function of one MCP as the other MCP angle is fixed for a cadaver hand [2] and for the ACT Hand.

contribution to movement. In essence, this is the same as changing from a forward to a reverse gear in a transmission. This change combined with the increase in moment arms with greater flexion noted above indicate that particular muscles become accessory or supporting muscles in the non-primary direction of motion. For instance, the FDP tendon seems to be able to assist in adduction/abduction in a bidirectional and non-uniform manner. In the regions where the moment arm is positive, increased tension in the muscle will contribute towards movement away from the centerline in the non-primary direction. Conversely, increased tension in the muscle where the moment arm is negative will contribute towards movement towards the centerline in the non-primary direction. Further studies into this phenomenon would show to what degree and in which patterns multiple muscles coactivate to modulate the fingertip force and stiffness.

Interestingly, the location of moment arm sign change is 


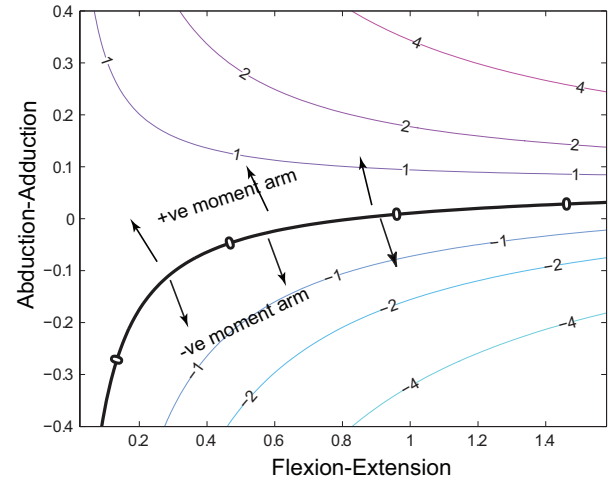

Fig. 7. Contours of FDP moment arm with respect to ab-ad angle. The zero contour is dependent on finger configuration defined by MCP angles.

non-constant. Figure 7 shows the zero level contour for the adduction/abduction moment arm of the FDP muscle. At a given flexion angle, the moment arm changes sign over adduction/abduction (i.e. the transmission changes direction from forward to reverse). It is interesting to note that this phenomenon occurs in the moment arm of the non-primary direction of motion.

Since the magnitudes of the moment arms generally increase with flexion angle (or in other words, the transmission ratio increases as the hand flexes), the variation in moment arm found above may be able to elucidate specific regions or hand poses where dexterity or force production ability is higher. Figure 8 shows variation in MCP joint

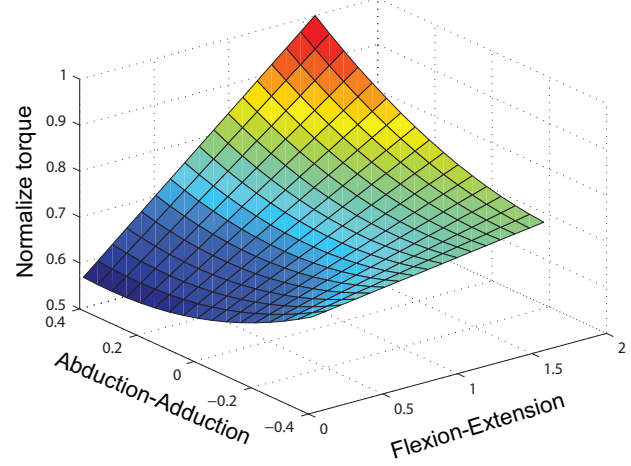

Fig. 8. MCP joint torque in the flexion (X) direction for various finger configuration as all six muscles are fully activated. The flexion torque is maximum when the finger is adducted and flexed.

torque in the flexion (around $\mathrm{X}$ axis) direction for various finger configurations as all six muscles are fully activated. This surface was created by using the relationship $\tau_{X}=$ $\sum-\left(R(i) \cdot f_{m}(i)_{\max }\right)$ where $\tau_{X}$ is the flexion torque, $R(i)$ is the non-constant moment arm for $i^{t h}$ muscle and $f_{m}(i)_{\max }$ is the maximum pull force generated by muscle $i$. The maximum force values were used from the literature [21]. This figure illustrates how the variations in moment arms affect the ability to produce force depending on joint angles. A constant moment arm assumption would lead to a flat surface for flexion torque. Our moment arm data suggests that the hand's ability to produce torque at the MCP joint increases as flexion and adduction angle increases. Physiologically, this allows the finger to produce the maximum palmar force when the finger is flexed and adducted.

By exploring the utility of moment arm variations in achieving hand movement control, we plan to further the understanding neuromuscular control of human hands. Also, we plan to investigate how moment arm variations affect hand dexterity and manipulability. Determination of moment arm variations would help in testing the viability of hand implants [24], [11]. It would be an important part of biomechanical model of finger dynamics [8], [18] and control [16]. So far, tendon arrangement and grasping abilities are analyzed with the assumption that moment arm is constant [14], [21], [5], [20]. Our results will help to update these studies to model anatomical hand motions. Moment arm determination will play an important role in developing next generation of hand animations [1] and simulations [28].

\section{REFERENCES}

[1] I. Albrecht, J. Haber, , and H.-P. Seidel. Construction and animation of anatomically based human hand models. In Eurographics/SIGGRAPH Symposium on Computer Animation, 2003.

[2] K. N. An, Y. Ueba, E. Y. Chao, W. P. Cooney, and R. I. Linscheid. Tendon excursion and moment arm of index finger muscles. Journal of Biomechanics, 16:419-425, 1983.

[3] Barrett. Barrett technology inc. http://www.barrett.com/robot/index.htm.

[4] A. Bicchi. Hands for dexterous manipulation and robust grasping: A difficult road toward simplicity. IEEE Transactions on Robotics and Automation, 16:652-662, 2000.

[5] A. Bicchi and D. Prattichizzo. Analysis and optimization of tendinous actuation for biomorphically designed robotic systems. Robotica, 18:23-31, 2000.

[6] K. B. Biggers, S. C. Jacobsen, and G. E. Gerpheide. Low level control of the utah/m. i. t. dexterous hand. In IEEE International Conference on Robotics and Automation, 1986.

[7] P. W. Brand, K. C. Cranor, and J. C. Ellis. Tendon and pulleys at the metacarpophalangeal joint of a finger. The Jounral of Bone and Joint Surgery, 57:779-784, 1975.

[8] N. Brook, J. M. nad M. Shoham, and J. Dayan. A biomechanical model of index finger dynamics. Med. Eng. Phys., 17:54-63, 1995.

[9] M. C. Carrozza, P. Dario, F. Vecchi, S. Roccella, M. Zecca, and F. Sebastiani. The cyberhand: on the design of cybernetic prosthetic hand intended to be interfaced to the peripheral nervous system. In Proceedings of IEEE/RSJ International Conference on Intelligent Robots and Systems, 2003.

[10] L. Y. Chang and Y. Matsuka. A kinematic thumb model for the act hand. In Proceedings of the IEEE International Conference on Robotics and Automation, 2006.

[11] B. DJ and S. BB. Metacarpophalangeal joint prostheses. a review of the clinical results of past and current designs. J Hand Surg, 20B:125136., 1995.

[12] A. G. Feldman. Functional tuning of the nervous system with control of movement or maintenance of a steady posture - ii. controllable parameters of the muscles. Biophysics, 11:565-578, 1966.

[13] N. Fowlera, A. Nicola, B. Condonb, and D. Hadleyc. Method of determination of three dimensional index finger moment arms and tendon lines of action using high resolution mri scans. Jounral of Biomechanics, 34:791-797, 2001.

[14] J. L. Fu and N. S. Pollard. On the importance of asymmetries in grasp quality metrics for tendon driven hands. In IEEE/RSJ International Conference on Intelligent Robots and Systems, 2006. 
[15] A. M. Herrmann and S. L. Delp. Moment arm and force-generating capacity of the extensor carpi ulnaris after transfer to the extensor carpi radialis brevis. The Journal of Hand Surgery, 24A:1083-1090, 1999.

[16] K. R. S. Holzbaur, W. M. Murray, and S. L. Delp. A model of the upper extremity for simulating musculoskeletal surgery and analyzing neuromuscular control. Annals of Biomedical Engineering, 33:829840, 2005.

[17] S. C. Jacobsen, D. F. Iversen, R. T. Knutti, and K. B. Biggers. Design of the utah/m. i. t. dexterous hand. In IEEE International Conference on Robotics and Automation, 1986.

[18] K.-H. Lee and K. H. Kroemer. A finger model with constant tendon moment arms. In PROCEEDINGS of the HUMAN FACTORS AND ERGONOMICS SOCIETY 37th ANNUAL MEETING, 1993.

[19] S. Narasimhan, D. M. Siegel, and J. M. Hollerbach. Condor: An architecture for controlling the utah-mit dexterous hand. IEEE Transactions on Robotics and Automation, 5:616-627, 1989.

[20] N. S. Pollard. Closure and quality equivalence for efficient synthesis of grasps from examples. The International Journal of Robotic Research, 6:595-613, 2004.

[21] N. S. Pollard and R. C. Gilbert. Tendon arrangement and muscle force requirements for humanlike force capabilities in a robotic finger. In Proceedings of IEEE International Conference on Robotics and Automation, 2002.

[22] RTAI. http://www.rtai.org.

[23] R. Shadmehr and M. A. Arbib. A mathematical analysis of the forcestiffness characteristics of muscles in control of a single joint system. Biological Cybenetics, 66:463-477, 1992.

[24] A. B. Swanson. Silicone rubber implants for replacement of arthritic or destroyed joints in the hand. Surg Clin North Am, 48:1113-1127, 1968.

[25] M. V. Weghe, M. Rogers, M. Weissert, and Y. Matsuoka. The act hand: Design of the skeletal structure. In Proceedings of the 2004 IEEE International Conference on Robotics and Automation, 2004.

[26] A.-P. C. Weiss, D. C. Moore, C. Infantolino, J. J. Crisco, E. Akelman, and R. D. McGovern. Metacarpophalangeal joint mechanics after 3 different silicone arthroplasties. The Journal of Hand Surgery, 29A:796-803, 2004.

[27] D. D. Wilkinson, M. V. Weghe, and Y. Matsuoka. An extensor mechanism for an anatomical robotic hand. In International Conference on Robotics \& Automation, 2003.

[28] J. William L. Buford, S. Koh, C. R. Andersen, and S. F. Viegas. Analysis of intrinsicextrinsic muscle function through interactive 3dimensional kinematic simulation and cadaver studies. The Journal of Hand Surgery, 6:1267-1275, 2005. 